\title{
A Shape Memory Alloy-Pendulum Damping System for Small Wild Goose Pagoda in Xi'an, China
}

\author{
Tao Yang ${ }^{1 *}$, Deming Liu ${ }^{1}$, Yang Liu ${ }^{1}$, Sheliang Wang ${ }^{2}$, Binbin $\mathrm{Li}^{2}$ \\ ${ }^{1}$ School of Urban Planning and Municipal Engineering, Xi'an Polytechnic University, Xi'an 710048, China \\ ${ }^{2}$ School of Civil Engineering, Xi'an University of Architecture and Technology, Xi'an 710055, China
}

Corresponding Author Email: yangtao@xpu.edu.cn

https://doi.org/10.18280/ijsse.100115

Received: 7 July 2019

Accepted: 21 November 2019

\section{Keywords:}

seismic response, shaking table test, shape memory alloy (SMA), pendulum damping

\begin{abstract}
This paper aims to develop a system to control the vibrations of ancient pagodas without damaging their original appearance. Taking the Small Wild Goose (SWG) Pagoda (Xi'an, Shaanxi Province) as the target structure, the authors designed a shape memory alloypendulum damping system (SMA-PDS) based on the theory of pendulum damping and the super elasticity of the SMA. To verify the effect of the SMA-PDS, a 1:10 scale model was created for the SWG Pagoda, and subjected to shaking table tests with and without the SMAPDS. The test results indicate that: the small and flexible system can be installed easily inside the pagoda to dampen seismic vibrations; the SMA-PDS greatly reduced the acceleration response at the top of the SWG Pagoda model; the reduction is particularly prominent $(32.5 \%)$, when the SMA wires had a pre-strain of 3\%; the SMA-PDS worked better in medium and large earthquakes than in small earthquakes. To sum up, this paper provides a novel damping system for seismic vibration in ancient pagodas, shedding new light on the energy dissipation, vibration control and reinforcement of similar ancient pagodas.
\end{abstract}

\section{INTRODUCTION}

Hailed as the oldest continuous civilization in the world, China boasts many well-preserved ancient buildings with high historical and cultural values. Pagodas are the tallest high-rise buildings in ancient China. These unique structures are rarely seen elsewhere in the world. The ancient pagodas carry rich humanistic values, and showcase the philosophy, aesthetics, history and culture of traditional China. In recent years, there is a growing awareness of the necessity to protect these architectural heritages [1-4].

Earthquakes pose a serious threat to the structural integrity of ancient pagodas. For example, the Famen Temple Pagoda (Fengxiang, Shaanxi Province), which was left unrepaired after in the 1976 Songpan-Pingwu earthquake, collapsed on the eastern side in a rainstorm in 1981. More than 30 ancient pagodas were damaged in different degrees during the 2008 Sichuan earthquake. Baita Temple Pagoda (Zhongjiang, Sichuan Province) collapsed from the top to the $10^{\text {th }}$ floor, while the Pen Pagoda (Yanting, Sichuan Province) tumbled down to the bottom. The 2015 Nepal earthquake destroyed many world cultural heritage sites in the region, including the Dharahara tower, a landmark in Nepal's capital of Kathmandu. Against this backdrop, it is imperative to enhance the seismic resistance of ancient pagodas and preserve their original appearance.

Many Chinese scholars have attempted to reduce the seismic impact on ancient pagoda. For instance, Yuan [5] established a dynamic response model for the Tiger Hill Pagoda (Suzhou, Jiangsu Province), and relied on the model to evaluate the structural reliability and damage state of the pagoda, laying the theoretical basis for dynamic research on similar ancient pagodas. $\mathrm{Lu} \mathrm{[6]} \mathrm{conducted} \mathrm{a} \mathrm{finite-element}$ analysis on Xingjiao Temple Pagoda (Xi'an, Shaanxi Province), calculated the seismic capacity of the masonry structure, and advised on the seismic fortification of the pagoda. Li et al. [7] designed an experiment and simulation, carried out a shaking table test on the damage criterion of the traditional masonry material in the pagoda, and simulated the damage evolution of the pagoda under the seismic waves of the 2008 Sichuan earthquake. However, the scale of the model is too small to fully reflect the structural features of the pagoda. Zhao et al. reinforced the Huaisheng Temple Pagoda (Guangzhou, Guangdong Province) with shape memory alloy (SMA), and proved that the reinforcement can reduce the seismic response of the pagoda through a shaking table test. Nevertheless, the reinforcement destroys the original appearance of the pagoda $[8,9]$.

In traditional seismic protection methods, local damages are allowed in structures to dissipate seismic energy. This means the structures must be sufficiently stiff, ductile and stable [1012]. These methods are clearly inapplicable to historical relics like ancient pagodas, which ought to be protected with minimum intervention.

Structural damping is a new method that has been proved effective in reducing the seismic response of structures [1316]. The most mature and popular damping technique is seismic energy dissipation, which is simple in structure, cheap in cost, and easy to use. More importantly, this technique will not alter the original appearance of the structure [17-21].

Taking the Small Wild Goose (SWG) Pagoda (Xi'an, Shaanxi Province) as the target structure, this paper designs a shape memory alloy-pendulum damping system (SMA-PDS) for ancient pagodas, based on the theory of pendulum damping and the super elasticity of the SMA. Next, a 1:10 scale model was created for the SWG Pagoda, according to the theory on 
structural similarity. Then, the authors compared the seismic responses of the SWG Pagoda models with and without the SMA-PDS through shaking table tests. The research results provide reference for seismic energy dissipation of ancient pagodas.

\section{INTRODUCTION TO THE SWG PAGODA}

The SWG Pagoda, named World Heritage site by the UNESCO in 2019, was built 1,300 years ago. For centuries, the pagoda has suffered from numerous natural disasters (e.g. earthquakes) and manmade damages. The resulting injuries continued to accumulate inside the pagoda. Currently, the SWG Pagoda has a poor seismic resistance. If another major earthquake occurs, the pagoda might be fatally damaged. To preserve the integrity of this heritage site, it is of paramount importance to enhance the seismic performance of the pagoda.

The SWG Pagoda (Figure 1) is an outstanding representative of Tang dynasty brick pagodas with dense eaves. The elegant shape and unique structure boast rich cultural and art values. The original pagoda was 15 floors high. The bottom 13 floors are preserved till this day. Currently, the pagoda is $43.3 \mathrm{~m}$ tall and $11.38 \mathrm{~m}$ wide on each side of the bottom. Each floor has a north-facing arched door and a south-facing arched door. The hollow body is surrounded by a single layer of walls with no column. Tourists can travel in the cylindrical inner space along a wooden staircase. The pagoda is wide and tall on the first floor, and gradually narrows and shortens in the upward direction. The roof is shaped like a natural dome.

The main structure of the pagoda was built by laying black bricks with staggered vertical joints. Field tests show that the compressive strength of the black bricks fell between 0.71 and $1.88 \mathrm{MPa}$. The bricks were bonded together by orange mud and quicklime. The two materials are sticky but not strong enough. In many places, the materials can be peeled off by bare hands. In addition to cracking, the exposed areas of the pagoda are severely weathered, while the interior is widely damaged. In 1965, the authority of heritage protection conducted a thorough repair of the pagoda. The repair mainly targeted the broken arched holes, the defected eaves, the damaged floors and the cracks on the body. In addition, the integrity of the pagoda was improved by adding reinforced concrete beams and slabs to the ceilings of the $2^{\text {nd }}, 5^{\text {th }}, 9^{\text {th }}$ and $13^{\text {th }}$ floors. The repaired internal structure is illustrated in Figure 2.

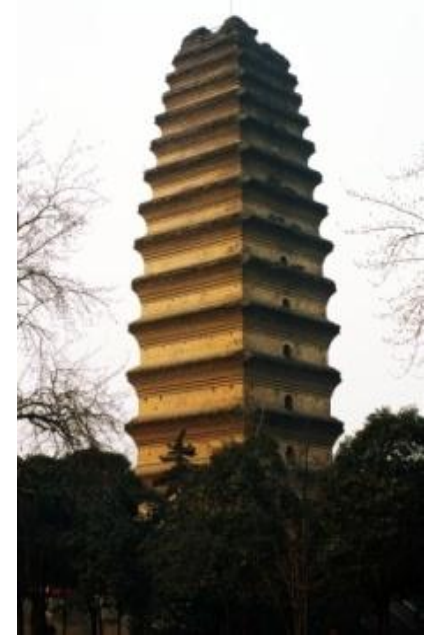

Figure 1. The current state of the SWG Pagoda

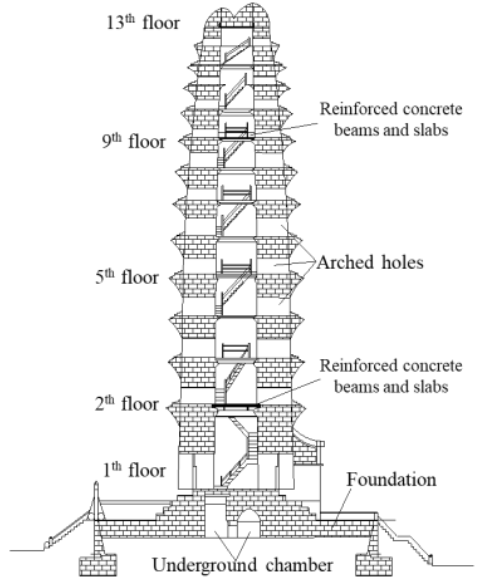

Figure 2. The internal structure of the SWG Pagoda

\section{DESIGN OF SMA-PDS}

Unlike modern buildings, the damping of ancient pagodas must follow the principles of protective repair of historical buildings. This paper combines the shape memory alloy (SMA) and pendulum damping into a composite damping system, denoted as the SMA-PDS. Considering the limited space inside the SWG Pagoda, the SMA wires were introduced as a damping element, so that the tuned mass damper could achieve desired damping effect with a small size. In this way, the SMA-PDS can effectively enhance the structure of the pagoda $[22,23]$.

As shown in Figure 3, the SMA-PDS mainly consists of mass vibrator, pendulum rod, one-way hinge, blocks, SMA wire, baffle, steering pulleys. The one-way hinge is attached to the upper end of the pendulum rod, and deployed perpendicular to the swing plane. There is a hole on the upper end of the pendulum rod, through which the spindle can move freely. The spindle is smooth in the middle and threaded at both ends, such that the pendulum rod can swing freely. The lower end of the pendulum rod is connected to the screw hole at the center of the mass vibrator. To facilitate mass adjustment, multiple mounting points are arranged symmetrically on the mass vibrator. There is an SMA wire on each side of the mass vibrator. On the one end, the wire is connected to a block through the baffle; on the other end, the wire, passing through a steering pulley, is connected to the wire rope via the adaptor, and thus fixed onto the target structure.

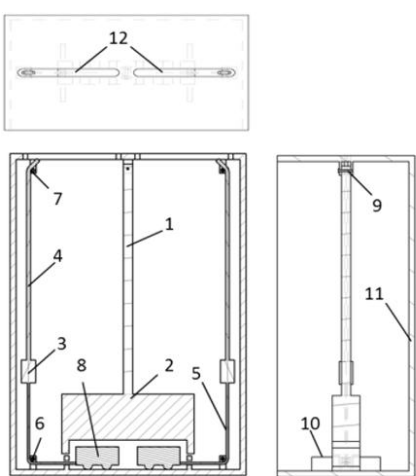

(a)Detail of construction

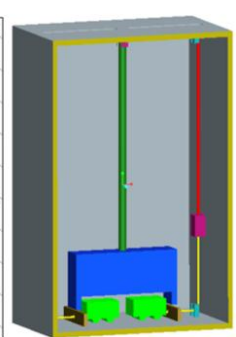

(b) $3 \mathrm{D}$ structure
1. Pendulum rod; 2. Mass vibrator; 3 . Wire conversion joint; 4 . Wire rope; 5 SMA wire; 6. Lower steering pulley; 7. Upper steering pulley; 8. Block; 9. One-way hinge; 10. Baffle; 11. Shell; 12. Channel

Figure 3. The structure of the SMA-PDS 
Figure 4 presents the connections between the SMA-PDS and the SWG Pagoda. Taking one damping cycle as an example, the working principle of the SMA-PDS was explained as follows: When the pendulum rod swings to the left, the right block is dragged along the horizontal slide, and the right SMA wire is dragged, creating a relative displacement $\Delta \varepsilon$. At this time, the SMA wire on the left is relaxed. Once the mass vibrator returns to the equilibrium position, the right SMA wire will become relaxed, forming a plump hysteresis curve. Through the damping cycle, the energy of the target structure is dissipated, while the inertial force of the mass vibrator spreads to the target structure via the wire rope. In this way, the vibration of the target structure is controlled, and its vibration response is attenuated.

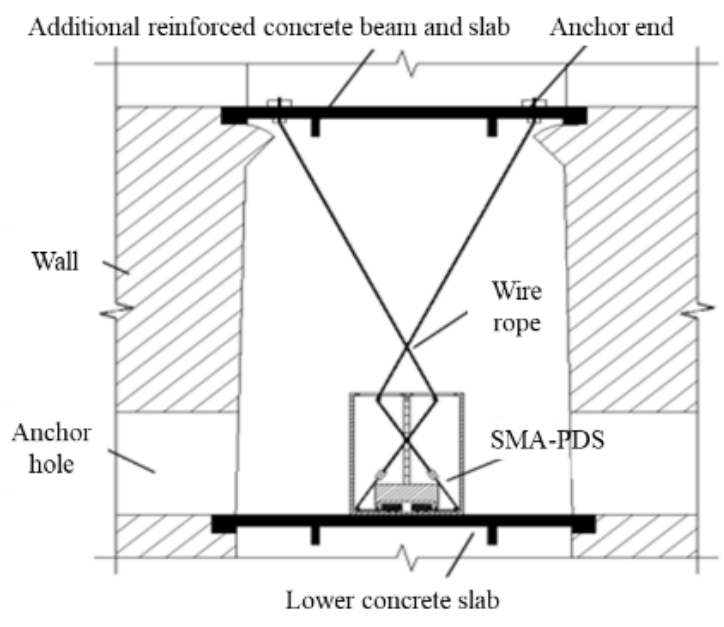

Figure 4. The connections between the SMA-PDS and the SWG Pagoda

\section{EFFECTIVENESS EXPERIMENTAL STUDY}

ANALYSIS

AND

\subsection{Effectiveness analysis}

Many scholars have investigated the interaction between tuned mass damper and the target structure. For instance, Ohsaki et al. [24] studied the phase of the tuned mass damper with a single degree-of-freedom (DOF) under the harmonic excitation, concluding that the energy transferred to the damper is the largest when the tuned mass damper lags behind the target structure by a phase difference of $90^{\circ}$ in relative displacement. Zhang et al. [25] suggested that the damping effect is the best when the displacement of the tuned mass damper has a phase difference of $180^{\circ}$ from the velocity of the target structure. Zhang proposed that, under the excitation of sine load, the tuned mass damper takes effect and reduces structural response, only if the phase difference between force and external excitation is $180^{\circ}$.

Drawing on the above results, this paper summarizes the relative positions between SMA-PDS and the SWG Pagoda into four cases (Figure 5), and also sums up the dynamic relationship between the equivalent force produced by the PDS and the pagoda structure (Figure 6).

In Figure 5, $x_{1}$ is the displacement vector of the SMA-PDS relative to the SWG Pagoda, $x_{2}$ is the displacement vector of the pagoda, and $\dot{x}_{2}$ is the velocity vector of the pagoda. Figure 6(a) corresponds to Cases (a) and (b) in Figure 5: if $x_{1} \times x_{2}<0$, the displacement of the SMA-PDS is opposite to the velocity of the pagoda, so is the equivalent force. Figure 6(b) corresponds to Cases (c) and (d) in Figure 5: if $x_{1} \times x_{2}>0$, the displacement of the SMA-PDS is in the same direction as the velocity of the pagoda, so is the equivalent force.

The above analysis shows that, the SMA-PDS cannot achieve the expected damping effect, unless the velocity response of the pagoda to external load has a certain phase difference from the displacement response of the mass vibrator in the SMA-PDS.

If the phase difference is $180^{\circ}$, both the displacement of the SMA-PDS and the equivalent force are opposite to the velocity of the pagoda. In this case, the SMA-PDS enjoys the best damping effect.

If the phase difference is $0^{\circ}$, both the displacement of the SMA-PDS and the equivalent force are in the same direction as the velocity of the pagoda. In this case, the SMA-PDS faces the worst damping effect, and even bolsters vibration.

This finding is very meaningful for damping effect analysis. The effectiveness of a damper can be evaluated in two simple steps: measuring the velocity of the target structure and the displacement of the damper; calculating the phase difference.

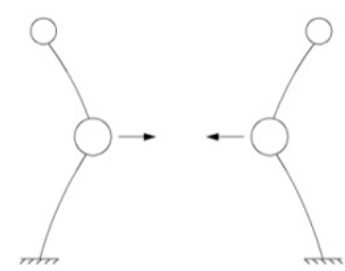

(a) $x_{1} \times x_{2}<0, x_{2} \times \dot{x}_{2}>0$

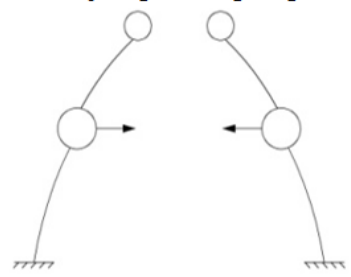

(c) $x_{1} \times x_{2}>0, x_{2} \times \dot{x}_{2}>0$

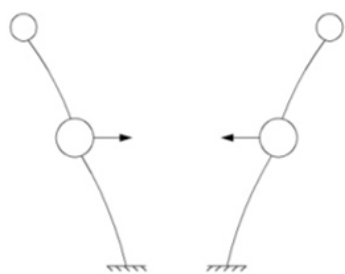

(b) $x_{1} \times x_{2}>0, x_{2} \times \dot{x}_{2}<0$

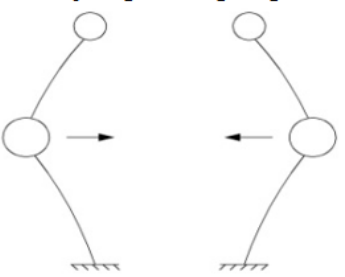

(d) $x_{1} \times x_{2}<0, x_{2} \times \dot{x}_{2}<0$
Figure 5. The relative positions between SMA-PDS and the SWG Pagoda

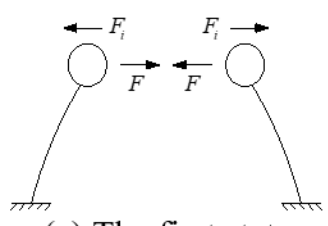

(a) The first state

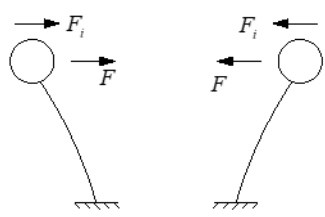

(b) The second state
Figure 6. Dynamic relationship between SMA-PDS and SWG Pagoda

\subsection{Experimental study}

Considering the effects from the pre-strain of SMA wire, shaking table tests were carried out on four SMA-PDS models with different pre-strains under sine excitation, with the aim to verify the damping effect of the SMA-PDS on the SWG Pagoda. The test conditions are listed in Table 1.

The SMA-PDS tests were performed on the WS-Z30-50 shaking table system in the Key Lab of Structure and Earthquake Resistance, Chinese Ministry of Education (Xi'an University of Architecture and Technology). The SMA-PDS 
was fixed onto the shaking table. During each test, displacement and acceleration sensors were deployed on the mass vibrator. Another displacement sensor was placed on the shaking table to measure the phase difference between the SMA-PDS and the table. Figure 7 is a photo of the SMA-PDS and the shaking table.

Table 1. SMA-PDS test conditions

\begin{tabular}{ccccc}
\hline $\begin{array}{c}\text { Test } \\
\text { number }\end{array}$ & $\begin{array}{c}\text { Mass } \\
(\mathbf{k g})\end{array}$ & $\begin{array}{c}\text { Pendulum } \\
\text { Length }(\mathbf{c m})\end{array}$ & $\begin{array}{c}\text { SMA diameter } \\
(\mathbf{m m})\end{array}$ & $\begin{array}{c}\text { Pre- } \\
\text { strain }\end{array}$ \\
\hline Z1 & & & & $0 \%$ \\
Z2 & 20 & 50 & 0.5 & $1 \%$ \\
Z3 & & & & $3 \%$ \\
Z4 & & & & $6 \%$ \\
\hline
\end{tabular}

The resonance method was adopted for the shaking table tests. However, the vibration frequency has a great impact on the SMA-PDS, and the phase of acceleration response on the table only affects the direction of the output force of the SMAPDS. Therefore, the main mode frequency of the SMA-PDS was determined without pre-strain on the SMA wires in

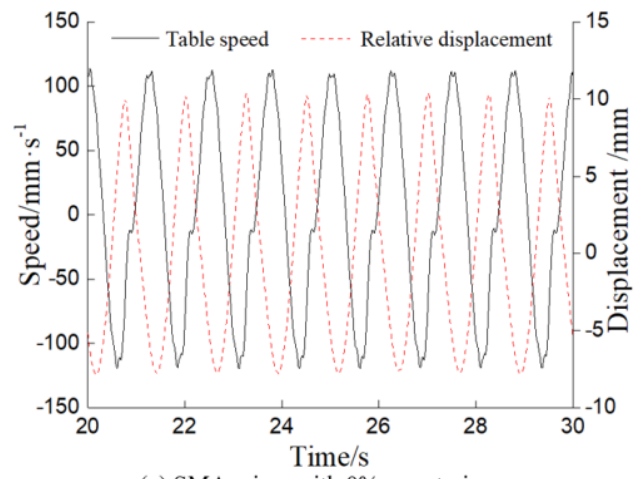

(a) SMA wires with $0 \%$ pre-strain

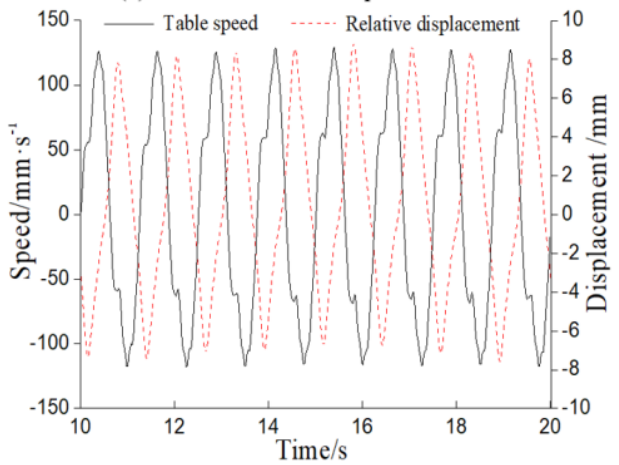

(c) SMA wires with $3 \%$ pre-strain advance. During the tests, the SMA-PDS was forced to vibrate at different frequencies near the main mode frequency. The input of forced vibration is a sine curve with the displacement of $8 \mathrm{~mm}$ and frequency of $0.8 \mathrm{~Hz}$. The test results were subjected to phase analysis, aiming to evaluate the damping effect of the SMA-PDS under the excitation of sine displacement. Figure 8 provides the time histories of SMAPDS displacement and the velocity of the shaking table. Table 2 lists the phase differences between the displacement and velocity.

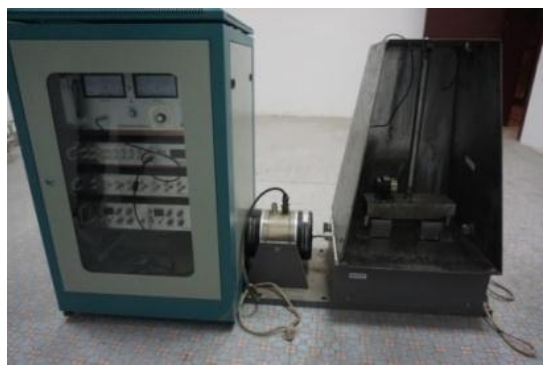

Figure 7. SMA-PDS and shaking table

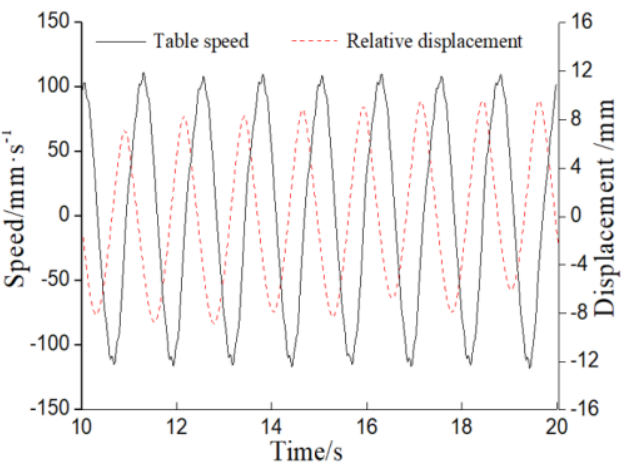

(b) SMA wires with $1 \%$ pre-strain

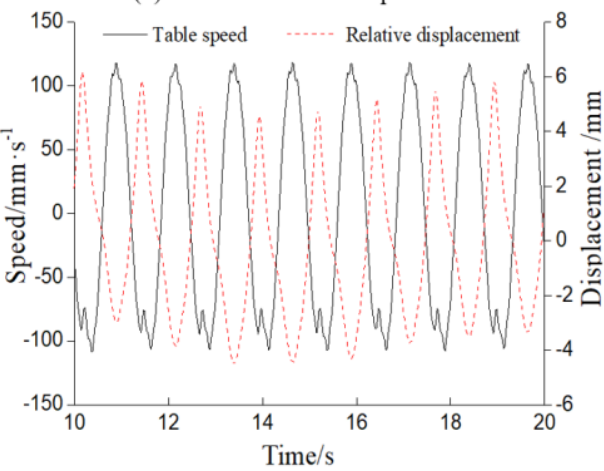

(d) SMA wires with $6 \%$ pre-strain

Figure 8. The time histories of displacement and velocity under sine excitation

Table 2. The phase differences between displacement and velocity

\begin{tabular}{ccccc}
\hline $\begin{array}{c}\text { Test } \\
\text { number }\end{array}$ & $\begin{array}{c}\text { Pendulum } \\
\text { Length } \\
(\mathbf{c m})\end{array}$ & $\begin{array}{c}\text { SMA } \\
\text { diameter } \\
(\mathbf{m m})\end{array}$ & $\begin{array}{c}\text { Pre- } \\
\text { strain }\end{array}$ & $\begin{array}{c}\text { Phase } \\
\text { difference }\end{array}$ \\
\hline Z1 & & & $0 \%$ & $152^{\circ}$ \\
Z2 & 50 & 0.5 & $1 \%$ & $149^{\circ}$ \\
Z3 & & & $3 \%$ & $160^{\circ}$ \\
Z4 & & & $6 \%$ & $164^{\circ}$ \\
\hline
\end{tabular}

As shown in Figure 8 and Table 2, the phase displacement of the SMA-PDS displacement and the velocity of the shaking table fell between $150^{\circ}$ and $180^{\circ}$. The mass vibrator moved in the opposite direction to the outer structure, and output a directional force to the latter. The results show that the SMAPDS has a good damping effect.

The phase differences of all SMA-PDS models were not greater than $180^{\circ}$, for the damping effect improves with the decrease in the frequency difference between the input sine wave and the SMA-PDS. To ensure the damping effect, the design parameters of the SMA-PDS should be optimized, such that the vibration frequency of the system is close to the natural vibration frequency of the target structure.

The mass of the mass vibrator was determined based on the assumed modal mass of the SWG Pagoda. The optimal frequency ratio and optimal damping ratio of the SMA-PDS were derived by the fixed point theory. 


\section{SHAKING TABLE TESTS ON SWG PAGODA MODEL WITH SMA-PDS}

\subsection{Model description}

According to the Code for Seismic Design of Buildings (GB 50011-2010), the location of the SWG Pagoda has a seismic fortification intensity of 8 and belongs to the $1^{\text {st }}$ earthquake group [26]. The basic seismic acceleration, site class and characteristic period $\mathrm{T}_{\mathrm{g}}$ were set to $0.2 \mathrm{~g}$, Class II, and $0.35 \mathrm{~s}$, respectively [27].

Considering the size and load capacity of the shaking table, the similarity coefficient $S_{1}$ was set to $1 / 10$. In the light of Buckingham $\mathrm{Pi}$ theorem and dimensional analysis, the similarity between the model and the pagoda was computed, and used to derive the similarity coefficients of our tests (Table 3) $[28-30]$.

Table 3. Similarity coefficients

\begin{tabular}{cccc}
\hline $\begin{array}{c}\text { Similar physical } \\
\text { quantity }\end{array}$ & Symbol & Formula & $\begin{array}{c}\text { Similarity } \\
\text { ratio }\end{array}$ \\
\hline Size & $S_{l}$ & Model $l /$ pagoda $l$ & 0.1 \\
Elastic modulus & $S_{E}$ & Model $E /$ pagoda $E$ & 1 \\
Mass & $S_{m}$ & Model $m /$ pagoda $m$ & 0.0036 \\
Density & $S_{\rho}$ & $S_{\rho}=S_{m} / S_{l}^{3}$ & 3.61 \\
Acceleration & $S_{\alpha}$ & $S_{a}=S_{E} S_{l}^{2} / S_{m}$ & 2.77 \\
Stress & $S_{\sigma}$ & $S_{\sigma}=S_{E} / S_{a}$ & 0.361 \\
Time & $S_{t}$ & $S_{t}=\sqrt{S_{l} / S_{a}}$ & 0.19 \\
Displacement & $S_{w}$ & $S_{w}=S_{l}$ & 0.1 \\
Velocity & $S_{v}$ & $S_{v}=\sqrt{S_{l} S_{a}}$ & 0.526 \\
Frequency & $S_{f}$ & $S_{f}=1 / S_{t}$ & 5.26 \\
\hline
\end{tabular}

In the design of the shaking table tests, the gravity distortion was reduced by increasing the artificial mass, thus ensuring the effectiveness of the structural force. The design of an undermanual quality model is adopted, in view of the size of the shaking table, as well as the bearing capacity and the internal conditions of the SWG Pagoda model. According to the similar relationship, a weight box containing steel blocks was set in the inner wall of the model. The additional weight is shown in Figure 9.

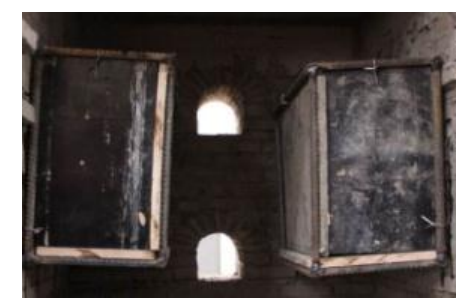

Figure 9. The additional weight

For the feasibility of our tests, the materials of the SWG Pagoda model were selected as follows: The pagoda model was made up of the processed grey bricks, which were produced in the1950s and 1960s. The bricks were cut into two specifications: the $110 \mathrm{~mm} \times 50 \mathrm{~mm} \times 25 \mathrm{~mm}$ bricks for pagoda body and the $110 \mathrm{~mm} \times 50 \mathrm{mmx} 10 \mathrm{~m}$ bricks for eaves. The bricks were bonded by quicklime, undisturbed loess and sticky rice pulp. The mix ratio of quicklime and undisturbed loess was 1:1. Figure 10 provides the photos of the materials and Figure
11 describes the dimensions and masonry structure of the model.
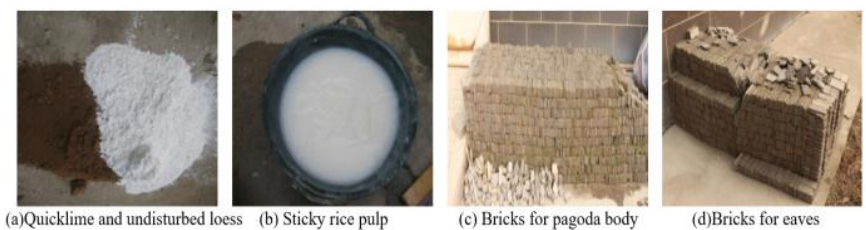

Figure 10. Materials of the model

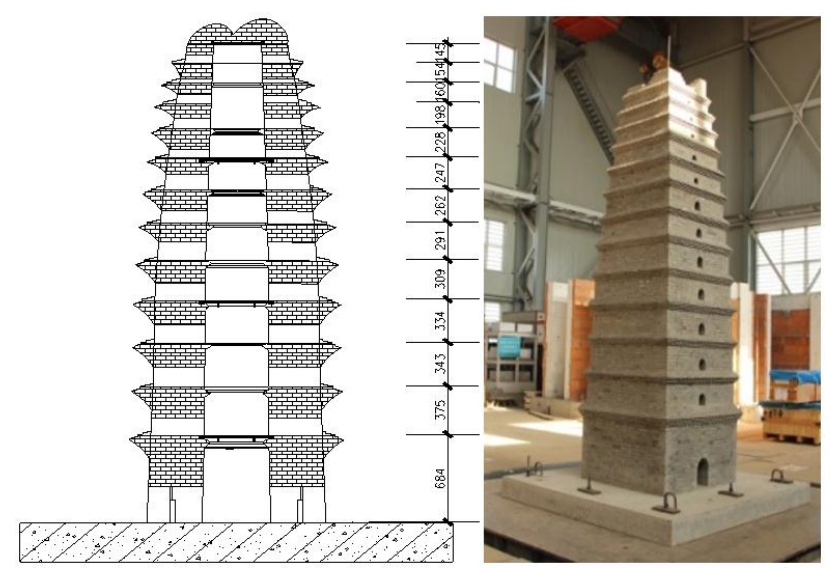

Figure 11. The SWG Pagoda model

\subsection{Test conditions}

The shaking table tests were conducted in the Key Lab of Structure Engineering and Earthquake Resistance, Chinese Ministry of Education (Xi' an University of Architecture and Technology), using a three-way 6DOF electro-hydraulic shaking table (MTS). The acceleration and displacement were collected by acceleration sensors and 891 displacement sensors (PCB). The test data were also collected by a data acquisition instrument (LMS).

Two natural seismic waves (El Centro and Wenchuan) and one artificial seismic wave (Shanghai wave) were adopted for our tests. The El Centro wave, the first seismic wave recorded by humans, was selected due to its rich spectral features. The Wenchuan wave was chosen, because the earthquake took place not far away from the location of the SWG Pagoda. The Shanghai wave was employed, because it was simulated on a soft soil foundation, while the SWG Pagoda was also built on such a foundation.

According to the seismic fortification intensity (8) of the SWG Pagoda, the shaking table tests explore the seismic responses of the pagoda model under 8-degree small earthquake, 8-degree medium earthquake and 8-degree strong earthquake. The tests were further divided into three levels: I, II and III, according to the setting of the SMA-PDS. In Level I tests, no damping system was adopted; In Level II tests, the SMA wires had a 3\% pre-strain; In Level III tests, the SMA wires had a $6 \%$ pre-strain. The working conditions of Level I tests are listed in Table 4. The working conditions of Level II and Level II tests are the same as those of Level I tests.

In the tests, the damping effect of the SMA-PDS was only considered along the direction of the arched hole, which is the most severely damaged place of the pagoda. The SMA-PDS could be arranged flexibly in any direction to meet the needs of actual application. 
Table 4. Conditions of Level I tests

\begin{tabular}{|c|c|c|c|c|}
\hline $\begin{array}{c}\text { Test } \\
\text { condition }\end{array}$ & $\begin{array}{c}\text { Test } \\
\text { number }\end{array}$ & $\begin{array}{c}\text { Seismic } \\
\text { wave }\end{array}$ & Direction & $\begin{array}{c}\text { Acceleration } \\
\text { peak (gal) }\end{array}$ \\
\hline \multirow{5}{*}{$\begin{array}{c}\text { 8-degree } \\
\text { small } \\
\text { earthquake }\end{array}$} & I 1 & $\begin{array}{l}\text { White } \\
\text { noise }\end{array}$ & \multirow{5}{*}{$X$} & 50 \\
\hline & I 2 & $\begin{array}{l}\text { El Centro } \\
\text { wave }\end{array}$ & & \\
\hline & I 3 & $\begin{array}{l}\text { Wenchuan } \\
\text { wave }\end{array}$ & & 200 \\
\hline & I 4 & $\begin{array}{l}\text { Shanghai } \\
\text { wave }\end{array}$ & & \\
\hline & I 5 & $\begin{array}{l}\text { White } \\
\text { noise }\end{array}$ & & 50 \\
\hline \multirow{4}{*}{$\begin{array}{c}\text { 8-degree } \\
\text { medium } \\
\text { earthquake }\end{array}$} & I 6 & $\begin{array}{l}\text { El Centro } \\
\text { wave }\end{array}$ & \multirow{4}{*}{$\mathrm{X}$} & \\
\hline & I 7 & $\begin{array}{l}\text { Wenchuan } \\
\text { wave }\end{array}$ & & 600 \\
\hline & I 8 & $\begin{array}{l}\text { Shanghai } \\
\text { wave }\end{array}$ & & \\
\hline & I 9 & $\begin{array}{l}\text { White } \\
\text { noise }\end{array}$ & & 50 \\
\hline \multirow{4}{*}{$\begin{array}{l}\text { 8-degree } \\
\text { earthquake }\end{array}$} & I 10 & $\begin{array}{l}\text { El Centro } \\
\text { wave }\end{array}$ & \multirow{4}{*}{$\mathrm{X}$} & \multirow{3}{*}{900} \\
\hline & I 11 & $\begin{array}{l}\text { Wenchuan } \\
\text { wave }\end{array}$ & & \\
\hline & I 12 & $\begin{array}{l}\text { Shanghai } \\
\text { wave }\end{array}$ & & \\
\hline & I 13 & $\begin{array}{l}\text { White } \\
\text { noise }\end{array}$ & & 50 \\
\hline
\end{tabular}

\subsection{Test phenomena}

The shaking table tests were conducted on the SWG Pagoda models under 8-degree small earthquake $(0.2 \mathrm{~g})$, 8-degree medium earthquake $(0.6 \mathrm{~g})$ and 8 -degree strong earthquake $(0.9 \mathrm{~g})$. The test phenomena of the SMA-PDS model with and without SMA-PDS were observed.

Under 8-degree small earthquake, Levels I, II and III tests exhibited similar responses. From the top bars of the model, the vibration response of the top layer was slightly greater under Wenchuan wave and Shanghai wave than that under El Centro wave. The tops bars vibrated significantly in Level I tests, and slightly in Levels II and III tests. No crack appeared on any other floor of the pagoda. Neither did cracks exist in the arched hole or eave on any floor. Overall, the model structure was intact, with a small seismic response.

When the peak acceleration of the seismic wave reached $0.4 \mathrm{~g}$, the vibration response of each test was more obvious than that of the small earthquake. The response was particularly prominent in the Level I test without SMA-PDS: the pagoda model had a significant vibration in the east-west direction, the amplitude of which was relatively large in the upper part; some dusts fell off the pagoda due to the vibration; meanwhile, cracks emerged near the arched holes and eaves in the upper part; fine horizontal cracks appeared on the eastern and western side at the foot of the pagoda. In general, the overall structure of the pagoda model was rather stable.

When the peak acceleration of the seismic wave reached $0.6 \mathrm{~g}$, the pagoda model showed clear differences in vibration responses under different working conditions. Under the same seismic wave, the Level II and III tests had a much smaller vibration amplitude of the top bars and less severe crack propagation than the Level I test. In the Level I test, the pagoda shook violently at the top; the cracks on the eastern and western sides at the foot spread towards the two sides and penetrated the entire wall on that floor; some sections were separated from the base. Near the arched holes in the north and south, local cracks emerged on the wall along the mortar joints, and propagated obliquely toward the holes, giving off brick breaking sounds on an occasionally basis. In the upper part of the pagoda, the eaves were seriously damaged, as some bricks became loose with fragments falling off; the arched holes in this part had many cracks, which were connected with the cracks on the nearby wall into penetrating cracks.

Under 8-degree strong earthquake, the Level II and III tests had a much smaller vibration amplitude of the top bars than the Level I test, achieving excellent damping effect. During Level I test, Wenchuan wave and Shanghai wave exerted the greatest impacts on the pagoda structure. Under the two waves, the pagoda model swayed greatly, giving off multiple sharp sounds of brick breakage. In the meantime, some bricks fell off the eaves in the upper part of the pagoda, while the pagoda body separated from the base on the western and eastern sides, before quickly returning to the original position. By contrast, the separation was not observed in the Level II and III tests, which target the pagoda model with the SMA-PDS. In these tests, the vibration amplitude of the upper part of the pagoda was much weaker than that in the Level I test; many bricks were broken on the wall, cracks were scattered and interlaced at the arched holes, and several eaves fell off; penetrating cracks were formed in the middle part of the pagoda, while the bricks were broken at the corners at the foot.

The above test phenomena indicate that, under seismic waves, the most unavertable parts of the SWG Pagoda include the foot, the arched holes in the middle, the eaves of each floor, and the top. The most severe damages were observed at the foot and the arched holes. The SMA-PDS greatly suppressed the seismic response and vibration amplitude of the upper part in the pagoda, and consumed the energy that may otherwise be dissipated by the pagoda body. In this way, the crack developed much slower on the pagoda body (Figure 12).

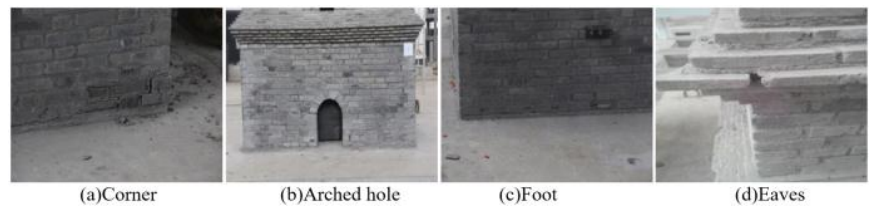

Figure 12. Test phenomena

\subsection{Test results and analysis}

\section{(1) Acceleration response}

The SWG Pagoda model was subjected to shaking table tests under El Centro wave, Wenchuan wave and Shanghai wave, respectively. For the lack of space, only the acceleration curves at the top (13th floor) of the pagoda under 8-degree strong earthquake are displayed in Figures 13-15. The peak accelerations in the middle of the pagoda ( $5^{\text {th }}$ floor) under the earthquake are recorded in Table 5.

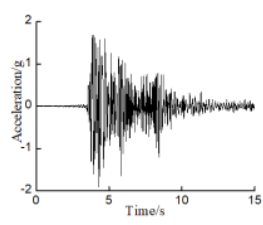

(a) Level I test

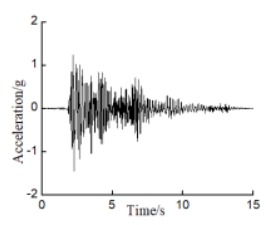

(b) Level II test

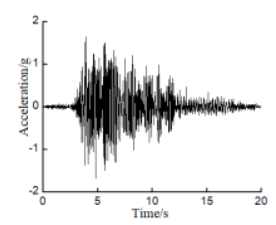

(c) Level III test
Figure 13. Acceleration response at the top under El Centro wave 


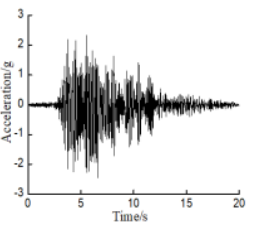

(a) Level I test

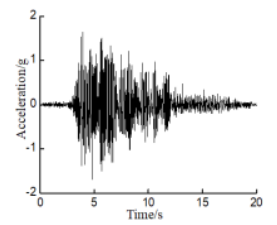

(b) Level II test

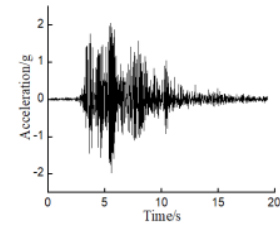

(c) Level III test
Figure 14. Acceleration response at the top under Wenchuan wave
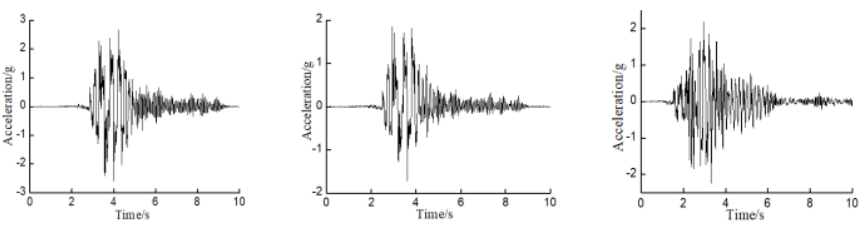

Figure 15. Acceleration response at the top under Shanghai wave

Table 5. Peak accelerations in the middle under 8-degree strong earthquake

\begin{tabular}{ccccccc}
\hline \multirow{2}{*}{$\begin{array}{c}\text { Working } \\
\text { condition }\end{array}$} & \multicolumn{2}{c}{$\begin{array}{c}\text { El Centro } \\
\text { wave }\end{array}$} & \multicolumn{2}{c}{$\begin{array}{c}\text { Wenchuan } \\
\text { wave }\end{array}$} & \multicolumn{2}{c}{$\begin{array}{c}\text { Shanghai } \\
\text { wave }\end{array}$} \\
\cline { 2 - 7 } & Max & Min & Max & Min & Max & Min \\
\hline Level I tests & 1.22 & -1.29 & 1.59 & -1.64 & 1.76 & -1.67 \\
Level II tests & 0.88 & -0.90 & 1.30 & -1.23 & 1.22 & -1.20 \\
Level III tests & 1.07 & -1.05 & 1.45 & -1.39 & 1.40 & -1.49 \\
\hline
\end{tabular}

As shown in Figures 13-15 and Table 5, the peak acceleration increased significantly to $1.76 \mathrm{~g}$ at the maximum in Level I tests.

Under the El Centro wave, peak accelerations at the middle and top of the pagoda in Level II tests were $29 \%$ and $27 \%$ smaller than those in Level I tests, respectively; those in Level III tests were $15.5 \%$ and $12.8 \%$ smaller than those in Level I tests, respectively.

Under the Wenchuan wave, peak accelerations at the middle and top of the pagoda in Level II tests were $21.8 \%$ and $26.8 \%$ smaller than those in Level I tests, respectively; those in Level III tests were $12.1 \%$ and $16.1 \%$ smaller than those in Level I tests, respectively.

Under the Shanghai wave, peak accelerations at the middle and top of the pagoda in Level II tests were $29.4 \%$ and $32.5 \%$ smaller than those in Level I tests, respectively; those in Level III tests were $18.1 \%$ and $14.5 \%$ smaller than those in Level I tests, respectively.

(2) Displacement response

The displacement curves at the top (13th floor) of the pagoda under 8-degree strong earthquake are displayed in Figures 16-18. The peak displacements in the middle of the pagoda (5th floor) under the earthquake are recorded in Table 6.

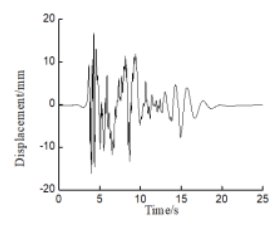

(a) Level I test

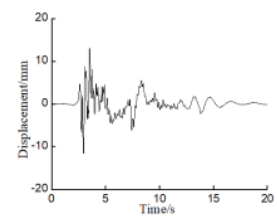

(b) Level II test

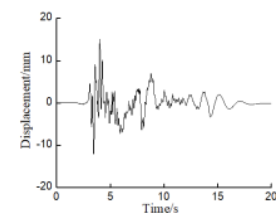

(c) Level III test
Figure 16. Displacement response at the top under El Centro wave

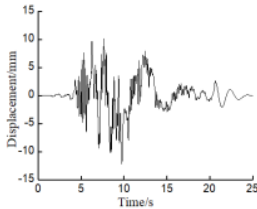

(a) Level I test (b) Level II test

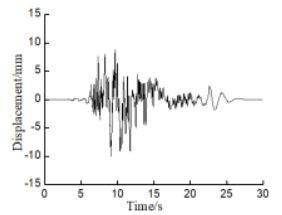

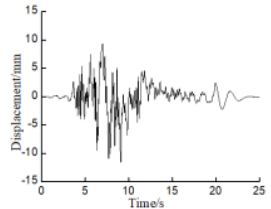

(c) Level III test
Figure 17. Displacement response at the top under Wenchuan wave

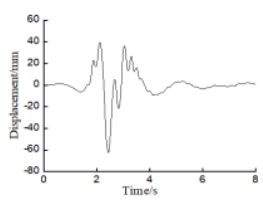

(a) Level I test

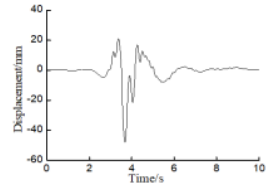

(b) Level II test

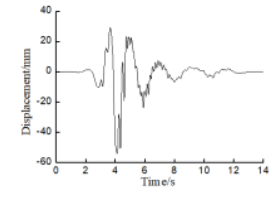

(c) Level III test
Figure 18. Displacement response at the top under Shanghai wave

Table 6. Peak displacements in the middle under 8-degree strong earthquake

\begin{tabular}{cccc}
\hline $\begin{array}{c}\text { Working } \\
\text { condition }\end{array}$ & $\begin{array}{c}\text { El Centro } \\
\text { wave }\end{array}$ & $\begin{array}{c}\text { Wenchuan } \\
\text { wave }\end{array}$ & $\begin{array}{c}\text { Shanghai } \\
\text { wave }\end{array}$ \\
\hline Level I tests & 2.13 .86 & 3.11 .65 & 4.45 .76 \\
Level II tests & 5.10 .22 & 6.9 .73 & 7.41 .36 \\
Level III tests & 8.13 .62 & 9.10 .76 & 10.43 .74 \\
\hline
\end{tabular}

As shown in Figures 16-18 and Table 6, the displacement responses in Level I tests were greater than those in Level II and III tests. Among them, the most obvious response appeared under the Shanghai wave. The results indicate that the SMA-PDS could effectively control the pagoda displacement under seismic waves.

Overall, the SMA-PDS showed an obvious damping effect under medium and strong earthquakes; the damping capacity slightly decreased, with the growth in the pre-strain of the SMA wires; despite the growth, the displacement response of the pagoda model was still controlled effectively.

It can also be seen that the SMA-PDS greatly reduced the acceleration response to the seismic waves. The average reduction was about $15 \%$. Under the SMA wires with $3 \%$ prestrain, the acceleration was decreased by an average of $>20 \%$, an evidence of excellent damping effect. However, if the prestrain continued to grow to $6 \%$, the damping effect actually weakened. This is because the $6 \%$ pre-strain is close to the ultimate tensile strain of the SMA wires, leaving a small deformation interval; therefore, the hysteresis loop of 3\% prestrain is smaller than that of $6 \%$ pre-strain, which weakens the capacity of energy dissipation. In spite of the above, the proposed SMA-PDS still achieved a good damping effect in the SWG Pagoda after the pre-strain climbed to $6 \%$.

The test results also demonstrate that the SMA-PDS worked better in medium and large earthquakes than in small earthquakes. The reason lies in the positive correlations between the following factors: the swing amplitude of the pendulum rod, the SMA deformation, the energy consumption and the damping effect.

\section{CONCLUSIONS}

Based on the principle similarity, this paper designs a 1:10 model of the SWG Pagoda, and performs shaking table tests 
on the model with and without the SMA-PDS. The following conclusions can be drawn from the tests:

(1) The authors designed an SMA-PDS for the special structure of the SWG Pagoda. The small and flexible system can be installed inside the pagoda without damaging its historical appearance. The system is also applicable to the energy dissipation, vibration control and reinforcement of similar ancient pagodas.

(2) The results of the shaking table tests were subjected to phase analysis. The analysis reveals that the mass vibrator moved in the opposite direction to the target structure, when the phase difference between the SMA-PDS displacement and table velocity fell between $150^{\circ}$ and $180^{\circ}$. In this case, the SMA-PDS could output an opposite force to the target structure, i.e. the system is effective in damping.

(3) The SMA-SPDS greatly reduced the acceleration response at the top of the SWG Pagoda model. The reduction is particularly prominent $(32.5 \%)$, when the SMA wires had a pre-strain of $3 \%$.

(4) The most vulnerable parts of the SWG Pagoda under seismic waves are along the direction of the arched hole. In our tests, the seismic waves were unidirectional, and the SMAPDS was also set in only one direction. In actual practices, the SMA-PDS could be placed in other directions to dampen the vibrations induced by seismic waves from various directions.

(5) The design of the SMA-PDS lives up to the principle of minimum intervention for seismic protection of ancient pagodas. The proposed system can effectively control the vibration response of the SWG Pagoda, and ancient pagodas of similar structure. The research results shed new light on seismic fortification of ancient brick pagodas.

\section{ACKNOWLEDGEMENTS}

This work was financially supported by the Shaanxi province natural science basic research program (2019JQ-578), Special research project of education department of Shaanxi province(18JK0332).

\section{REFERENCES}

[1] Wang, G.L., Wu, G.K., Wang, Y.M., Guo, M., Zhao, J.H., Gao, C. (2018). Deformation monitoring of ancient pagoda with multi-source data. Journal of Geoinformation $\quad$ Science, 20(4): 496-504. https://doi.org/10.12082/dqxxkx.2018.170446

[2] Lam, F., He, M., Yao, C. (2008). Example of traditional tall timber buildings in China-the Yingxian pagoda. Structural Engineering International, 18(2): 126-129. https://doi.org/10.2749/101686608784218743

[3] Song, X., Wu, Y., Li, K., Jin, L., Chen, F., Liu, X., Dou, X. (2019). Mechanical behavior of a Chinese traditional timber pagoda during construction. Engineering Structures, 196:

109302 https://doi.org/10.1016/j.engstruct.2019.109302

[4] Xie, Q., Xu, D., Wang, Y., Zhang, L., Xin, R. (2019). Seismic behavior of brick masonry walls representative of ancient Chinese pagoda walls subjected to in-plane cyclic loading. International Journal of Architectural Heritage, $1-13$. https://doi.org/10.1080/15583058.2019.1674943

[5] Yuan, J.L. (2018). Analytical method on horizontal earthquake action of ancient masonry pagoda. Earthquake Engineering and Engineering Dynamics, 38(2):

$18-27$. https://doi.org/10.13197/j.eeev.2018.02.18.yuanj1.003

[6] Lu, J.L. (2016). Damage and dynamic characteristics analysis of a solid structure pagoda. Mechanics in Engineering, $\quad 38(5)$ : 560-566. https://doi.org/10.6052/1000-0879-16-064

[7] Li, T., Wang, S., Yang, T. (2018). Experiment and simulation study on vibration control of an ancient pagoda with damping devices. International Journal of Structural Stability and Dynamics, 18(10): 1850120. https://doi.org/10.1142/S0219455418501201

[8] Zhao, X., Wang, S.L., Zhou, F.L., Huang, X.Y., Zhao, X.C. (2011). Shaking table tests for ancient pagoda model structure based on shape memory alloy actuating devices. Journal of Vibration and Shock, 30(11): 219223. https://doi.org/10.3969/j.issn.10003835.2011.11.044

[9] Lu, X., Zhou, Y., Lu, W. (2007). Shaking table model test and numerical analysis of a complex high - rise building. The Structural Design of Tall and Special Buildings, 16(2): 131-164. https://doi.org/10.1002/tal.302

[10] Jara, J.M., Olmos, B.A., Martínez, G. (2018). Strength and stiffness parameters of energy dissipation devices for the seismic protection of building on soft soils. Bulletin of Earthquake Engineering, 16(9): 4297-4313. https://doi.org/10.1007/s10518-018-0312-1

[11] Ahmad, B.H., Marco, V., Gabriele, M. (2019). Effectiveness of different base isolation systems for seismic protection: Numerical insights into an existing masonry bell tower. Soil Dynamics and Earthquake Engineering, 125 : 105752 . https://doi.org/10.1016/j.soildyn.2019.105752

[12] Marinković, M., Butenweg, C. (2019). Innovative decoupling system for the seismic protection of masonry infill walls in reinforced concrete frames. Engineering Structures, $197(2019)$ : 109435. https://doi.org/10.1016/j.engstruct.2019.109435

[13] Ismail, M. (2019). An elastoplastic bracing system for structural vibration control. Engineering Structures, 200: 109671. https://doi.org/10.1016/j.engstruct.2019.109671

[14] Saha, S., Debbarma, R. (2017). Dynamic response control of structures using liquid column vibration absorber: an experimental study. International Journal of Advanced Structural Engineering, 9(3): 269-275. https://doi.org/10.1007/s40091-017-0163-Z

[15] Lu, Z., Wang, Z., Zhou, Y., Lu, X. (2018). Nonlinear dissipative devices in structural vibration control: A review. Journal of Sound and Vibration, 423: 18-49. https://doi.org/10.1016/j.jsv.2018.02.052

[16] Ying, Z., Ni, Y. (2017). Advances in structural vibration control application of magneto-rheological viscoelastomer. Theoretical and Applied Mechanics Letters, 7(2): 61-66. https://doi.org/10.1016/j.taml.2017.01.003

[17] Li, Z., Shu, G., Huang, Z. (2019). Proper configuration of metallic energy dissipation system in shear-type building structures subject to seismic excitation. Journal of Constructional Steel Research, 154: 177-189. https://doi.org/10.1016/j.jcsr.2018.11.032

[18] Benavent-Climent, A., Oliver-Saiz, E., Donaire-Avila, J. (2015). New connection between reinforced concrete building frames and concentric braces: Shaking table 
tests. Engineering Structures, 96: 7-21. https://doi.org/10.1016/j.engstruct.2015.03.023

[19] Kandemir-Mazanoglu, E.C., Mazanoglu, K. (2017). An optimization study for viscous dampers between adjacent buildings. Mechanical Systems and Signal Processing, 89: 88-96. https://doi.org/10.1016/j.ymssp.2016.06.001

[20] Bagheri, S., Rahmani-Dabbagh, V. (2018). Seismic response control with inelastic tuned mass dampers. Engineering Structures, 172: 712-722. https://doi.org/10.1016/j.engstruct.2018.06.063

[21] Al-Fahdawi, O.A., Barroso, L.R., Soares, R.W. (2019). Semi-active adaptive control for enhancing the seismic performance of nonlinear coupled buildings with smooth hysteretic behavior. Engineering Structures, 191: 536548. https://doi.org/10.1016/j.engstruct.2019.04.078

[22] Ok, S.Y., Song, J., Park, K.S. (2008). Optimal performance design of bi-tuned mass damper systems using multi-objective optimization. KSCE Journal of Civil Engineering, 12(5): 313-322. https://doi.org/10.1007/s12205-008-0313-8

[23] Billah, A.M., Alam, M.S. (2016). Plastic hinge length of shape memory alloy (SMA) reinforced concrete bridge pier. Engineering Structures, 117: 321-331. https://doi.org/10.1016/j.engstruct.2016.02.050

[24] Ohsaki, M., Tsuda, S., Hasegawa, T. (2016). Parameter optimization of tetrahedral tuned mass damper for threedirectional seismic response reduction. Engineering
Structures,

126:

667-674.

https://doi.org/10.1016/j.engstruct.2016.08.014

[25] Zhang, J.P., Yu, Q.C., Zhou, F.L. (2000). Two theoretical problems in structural vibration control. Earthquake Engineering and Engineering Vibration, 20(1): 125-129. https://doi.org/10.3969/j.issn.1000-1301.2000.01.019

[26] GB50011-2010 (2010). Code for seismic design of buildings. Beijing: China Architecture \& Building Press.

[27] JGJ/T 101-2015 (2015). Specification for Seismic Test of Building. Beijing: China Architecture \& Building Press.

[28] Xue, J., Zhao, X., Zhang, F., Xu, D., Hu, X., Ma, L. (2020). Shaking table tests on seismic behavior of the underground loess cave of earth building of traditional dwellings. Engineering Structures, 207: 110221. https://doi.org/10.1016/j.engstruct.2020.110221

[29] Zhou, T., Liu, B. (2019). Experimental study on the shaking table tests of a modern inner-reinforced rammed earth structure. Construction and Building Materials, 203: 567-578. https://doi.org/10.1016/j.conbuildmat.2019.01.070

[30] Guan, Z., Li, J., Guo, W., Qu, H. (2019). Design and validation of a shaking-table test model on a long-span cable-stayed bridge with inverted-Y-shaped towers. Engineering Structures, 201: 109823. https://doi.org/10.1016/j.engstruct.2019.109823 